\title{
Enhanced Temporal Response Properties of Anteroventral Cochlear Nucleus Neurons to Broadband Noise
}

\author{
Dries H. G. Louage, Marcel van der Heijden, and Philip X. Joris \\ Laboratory of Auditory Neurophysiology, Medical School, K. U. Leuven, B-3000 Leuven, Belgium
}

\begin{abstract}
Compared with auditory nerve (AN) fibers, trapezoid body (TB) fibers of the cat show enhanced synchronization to low-frequency tones. This phenomenon probably contributes to the high temporal resolution of binaural processing. We examined whether enhanced synchronization also occurs to sustained broadband noise. We recorded responses to a reference Gaussian noise and its polarity-inverted version in the TB of barbiturate-anesthetized cats. From these we constructed shuffled autocorrelograms (SACs) and quantified spike timing by measuring the amplitude and width of their central peak.

Many TB fibers with low characteristic frequency (CF) showed SACs with higher and narrower central peaks than ever observed in the AN, indicating better consistency and precision of temporal coding. Larger peaks were also observed in TB fibers with high CF, but this was mostly caused by higher average firing rates, resulting in a larger number of coincident spikes across stimulus repetitions. The results document monaural preprocessing of the temporal information delivered to binaural nuclei in the olivary complex, which likely contributes to the high sensitivity to interaural time differences.
\end{abstract}

Key words: synchronization; ventral cochlear nucleus; auditory nerve; noise; autocorrelation; phase-locking; bushy cells

\section{Introduction}

A remarkable property of peripheral auditory neurons is the extent to which they encode temporal features of the acoustic waveform (Galambos and Davis, 1943; Kiang et al., 1965; Rose et al., 1967; Johnson, 1980). Temporal features are coded through phase-locking, i.e., spike discharges are locked to certain events of the waveform. The accurate neural coding of these features is required for the exquisite sensitivity to interaural time differences (ITDs) that is observed behaviorally (Klump and Eady, 1956) and physiologically (Rose et al., 1966; Goldberg and Brown, 1969; Yin et al., 1986; Shackleton et al., 2003; Hancock and Delgutte, 2004).

The superior olivary complex (SOC) plays a key role in the binaural comparison of temporal features at the two ears. Many cells in the SOC are sensitive to ITDs in the stimulus waveform, either in its fine structure or its envelope (Goldberg and Brown, 1969; Moushegian et al., 1975; Yin and Chan, 1990; Finlayson and Caspary, 1991; Joris and Yin, 1995; Spitzer and Semple, 1995; Batra et al., 1997). Naturally, good ITD sensitivity in the SOC demands accurate temporal waveform coding by its monaural inputs. The SOC receives its dominant input from bushy cells of the anteroventral cochlear nucleus (AVCN). Joris et al. (1994a) reported that bushy cells in the cat show enhanced synchronization to short low-frequency tones when compared with auditory nerve (AN) fibers. This enhanced synchronization reflects AVCN processing of AN input and presumably serves to optimize ITD

Received Nov. 19, 2004; revised Dec. 28, 2004; accepted Jan. 3, 2005.

This work was supported by grants from the Fund for Scientific Research-Flanders (G.0083.02) and K. U. Leuven Research Fund (0T/10/42). M.v.d.H. was supported by a K. U. Leuven fellowship (F/00/92).

Correspondence should be addressed to Philip X. Joris, Laboratory of Auditory Neurophysiology, Campus GHB, 0\&N bus 801, B-3000 Leuven, Belgium. E-mail: Philip.Joris@med.kuleuven.ac.be.

D01:10.1523/JNEUROSCI.4742-04.2005

Copyright $\odot 2005$ Society for Neuroscience $\quad$ 0270-6474/05/251560-11\$15.00/0 sensitivity at low frequencies in the SOC (Joris et al., 1994a; Brughera et al., 1996; Fitzpatrick et al., 1997; Fitzpatrick and Kuwada, 2001; Marsalek, 2001).

Synchronization enhancement in the AVCN has been analyzed exclusively in terms of vector strength to pure tones (Blackburn and Sachs, 1989; Joris et al., 1994a; Paolini et al., 2001; Köppl and Carr, 2003) and sinusoidally amplitude-modulated tones (Frisina et al., 1990; Rhode and Greenberg, 1994; Joris and Yin, 1998). Here, we seek to generalize the study of synchronization to a wider class of stimuli. Recently, we introduced shuffled autocorrelograms (SACs) (Joris, 2003) to quantify synchronization to any stimulus. This approach allows a more general characterization of temporal behavior than periodic stimuli and Fourier analysis (Louage et al., 2004). Moreover, the SAC is based on the counting of coincidences, which resembles the type of neural processing generally assumed to underlie ITD sensitivity (Goldberg and Brown, 1969; Yin and Chan, 1990).

In the present study, we use SACs to analyze the responses of AVCN axons in the trapezoid body (TB) to broadband noise. The results for the TB are compared with published AN data obtained in the same species, with the same stimuli, recording procedures, and analysis parameters (Louage et al., 2004). Our main finding is that low-frequency TB fibers respond to broadband noise with better precision and consistency than AN fibers.

\section{Materials and Methods}

Animal preparation. Cats with normal eardrums and middle ears were anesthetized with a mixture of acepromazine $(0.2 \mathrm{mg} / \mathrm{kg})$ and ketamine $(20 \mathrm{mg} / \mathrm{kg})$. A venous cannula allowed infusion of Ringer's solution and sodium pentobarbital at doses sufficient to maintain an areflexic state. A tracheostoma was made, and a laryngopharyngectomy was performed. The basioccipital bone was exposed after resection of the prevertebral muscles. The pinnas were removed, and the bullas were exposed and 


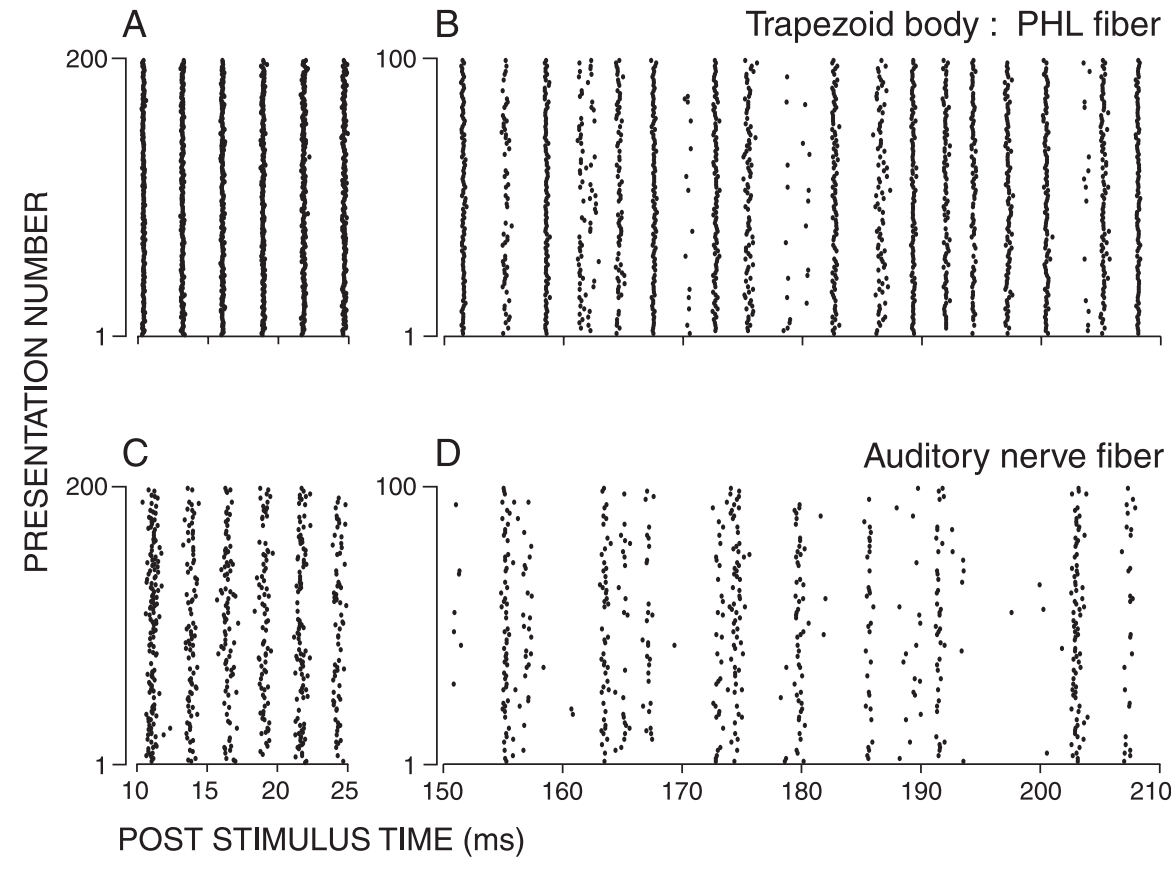

Figure 1. Dot rasters of responses to pure tones at the $C F(A, C)$ and to broadband noise $(B, D)$ for a PHL trapezoid body fiber (top) and an AN fiber (bottom). CF, threshold, and SR were $350 \mathrm{~Hz}, 23 \mathrm{~dB} \mathrm{SPL}$, and 90 spikes/s (top) and $379 \mathrm{~Hz}$, $39 \mathrm{~dB} \mathrm{SPL}$, and 57 spikes/s (bottom). Sound levels (dB SPL) were $40(A), 60(B), 60$ (C), and 80 (D).

vented with a 30 -cm-long polyethylene tube (inner diameter, $0.9 \mathrm{~mm}$ ). The animal was placed in a double-walled soundproof room (Industrial Acoustics Company, Niederkrüchten, Germany). The TB was exposed by drilling a longitudinal slit as close as possible to the medial wall of the bulla and $\sim 3-5 \mathrm{~mm}$ rostral to the jugular foramen. A micromanipulator was used to support a hydraulic microdrive (Trent Wells, Coulterville, $\mathrm{CA}$ ). Glass micropipettes, filled with $3 \mathrm{M} \mathrm{NaCl}$ or $\mathrm{KCL}$, were positioned in the TB under visual control, just lateral or medial to the rootlets of the abducens nerve. The angle of penetration ranged from 0 to $30^{\circ}$ mediolaterally relative to the midsagittal plane. After placing the electrode in the $\mathrm{TB}$, the basioccipital bone was covered with warm $3 \%$ agar.

Hardware and software. Dynamic phones (supertweeter; Radio Shack, Forth Worth, TX) were connected with tygon tubing to Teflon earpieces that fit tightly in the transversely cut ear canals. Custom software, run within Matlab (The MathWorks, Natick, MA) on a personal computer, was used to calculate the stimuli and control the digital hardware (Tucker-Davis Technologies, Alachua, FL). All stimuli were computed at a sample rate of $125 \mathrm{kHz}$ and passed through an antialiasing filter with a cutoff of $60 \mathrm{kHz}$. The transfer function of the closed acoustic assembly was obtained via a probe whose tip was placed within $2 \mathrm{~mm}$ of the eardrum and that was coupled to a $1 / 2$ inch $(12.7 \mathrm{~mm})$ condensor microphone and conditioning amplifier (Bruel \& Kjaer, Nærum, Denmark). All stimuli were compensated for this transfer function, and the stimuli were specified in sound pressure level (SPL) ( $\mathrm{dB}$ re $20 \mu \mathrm{Pa})$. The neural signal was amplified and filtered $(300 \mathrm{~Hz}$ to $3 \mathrm{kHz}$ ) (DAM 80; World Precision Instruments, Sarasota, FL), and spikes were converted to standard pulses with a custom-built peak-picker (Carney and Yin, 1988) or, in the earliest experiments, with a level discriminator (DIS-1; BAK Electronics, Germantown, MD). These pulses were time stamped to an accuracy of $1 \mu$ s (ET-1; Tucker-Davis Technologies).

Stimuli. Search stimuli consisted of noise bursts (duration $300 \mathrm{~ms}$, repeated every $500 \mathrm{~ms}, 70 \mathrm{~dB}$ SPL, bandwidth $40 \mathrm{kHz}$ ), delivered to both ears. When a single unit was isolated, the excitatory ear was determined. Binaural neurons were occasionally encountered but are not considered in this report. Spontaneous rate (SR), minimum rate threshold, and characteristic frequency (CF) of single fibers were determined with an automated threshold-tracking program. Short tone bursts at CF (duration $25 \mathrm{~ms}$, repeated every $100 \mathrm{~ms}, 200$ repetitions, rise-fall time $2.5 \mathrm{~ms}$, starting in sine phase) were then presented at increasing SPL in $10 \mathrm{~dB}$ steps. Various response metrics were displayed on-line.

A frozen noise waveform (Gaussian broadband noise, $0.1-30 \mathrm{kHz}, 1000 \mathrm{~ms}$, repeated every $1200 \mathrm{~ms}, 10$ repetitions) was digitally synthesized using the inverse Fast Fourier Transform method. It was presented from 10 to $90 \mathrm{~dB}$ SPL in $10 \mathrm{~dB}$ steps to obtain a rate-level curve. The same noise was then presented at least 50 times at $70 \mathrm{~dB}$ SPL and when possible also at other levels. A second series of responses to the same noise waveform at the same SPLs was also obtained, the only difference being that the waveform was inverted in polarity. Inverting the polarity corresponds to a $180^{\circ}$ phaseshift of all frequency components. Because the normalized correlation coefficient of the reference waveform and its inverted version is -1 , we refer to the two waveforms as reference noise and anticorrelated noise.

Peristimulus time histogram classification. Fibers were classified into different categories based on the shape of their peristimulus time histogram (PSTH) (binwidth $0.1 \mathrm{~ms}$ ) to short pure tone bursts at $\mathrm{CF}$, following the qualitative scheme first developed by Pfeiffer (1966) and elaborated by others. "Primary-like" (PL) PSTHs resemble those of AN fibers, with an initial peak followed by a monotonic decline in rate to a steady-state response. "Primary-likewith-notch" $\left(\mathrm{PL}_{\mathrm{N}}\right)$ fibers have PSTHs with a brief notch following the initial peak. This notch is difficult to detect for fibers that phase-lock and have a CF of $<1200 \mathrm{~Hz}$ (Smith et al., 1993), and such fibers were therefore classified as "phase-lockers" (PHLs). PSTHs with regularly spaced peaks of discharge whose period was unrelated to the stimulus waveform, were classified as "chopper" (CHOP). We sometimes obtained PSTHs whose initial peak had an unusually long latency (>11 ms): these PSTHs were classified as "unusual." Fibers for which no responses to short tone bursts were available were classified as "no PSTH."

Synchronization to tones. From the short pure tone responses, vector strength $(R)$ was determined; $R$ is the Fourier component of the PSTH at the stimulus frequency, normalized by the total number of spikes (Goldberg and Brown, 1969). The vector strength was calculated over an analysis window of $10-25 \mathrm{~ms}$ relative to the stimulus onset to eliminate the onset response, which was not always in phase with the sustained response. Significance $(p<0.001)$ of phase-locking was evaluated with the Rayleigh test (Mardia and Jupp, 2000).

Temporal properties to broadband noise. We constructed SACs with binwidths of $50 \mu$ s over an analysis window of 50-1000 ms relative to the stimulus onset from the responses to broadband noise (Joris, 2003). The construction of SACs involves four steps. First, a set of spike trains was collected by repeatedly presenting the same stimulus. Second, all possible pairs of spike trains from different presentations were listed. Third, for each pair, forward time intervals between all spikes of the first spike train and all spikes of the second spike train were measured. Fourth, all intervals from all listed pairs were tallied, yielding the shuffled all-order interval histogram, i.e., the SAC. The number of intervals in each bin of the SAC depends on recording parameters (number of presentations $N$, and stimulus duration $D$ ) and firing properties of the fiber. We scale the ordinate of the SAC in two ways. When the effect of recording parameters is factored out by dividing through $N(N-1) D$, the bin values are in units of coincidences/s, and we will refer to the maximal bin value as the maximal coincidence rate $\left(\mathrm{CR}_{\max }\right)$; in SACs this maximum always occurs at delay 0 . SACs can also be plotted on a normalized scale by dividing through $N(N-1) \Delta \tau r^{2} D$. This factor eliminates the effect of average firing rate $r$, number of presentations $N$, choice of bin width $\Delta \tau$, and stimulus duration $D$ (for a detailed description of this metric, see Louage 
et al., 2004). This scaling yields dimensionless bin values, and we will refer to the maximal $y$ value as the correlation index (CI). With this scale, uncorrelated spike trains result in a value of 1 .

Fibers with CFs in the range of pure-tone phase-locking show an oscillatory SAC (see Results). To quantify the frequency of this oscillation, we applied a $30 \mathrm{~ms}$ Hanning window with its maximum at delay zero, applied zero-padding to extend the SAC to $2^{17}$ points, and then calculated the magnitude spectrum with a Fast Fourier Transform algorithm. Magnitude spectra of SACs always had a primary peak at low frequencies with a maximum at DC; this peak (all components $<150 \mathrm{~Hz}$ ) was ignored. We refer to the dominant frequency (DF) of the SAC as the frequency at which a secondary peak in the magnitude spectrum occurred.

Responses to reference and anticorrelated noise were used to construct cross-stimulus autocorrelograms (XACs) (Joris, 2003). The construction of XACs differs from that of SACs in that the spike trains of each pair are obtained with a different stimulus: the reference and anticorrelated noise. XACs of fibers with CF in the range of pure-tone phase-locking oscillate in anti-phase with SACs (see Results and Louage et al., 2004). XACs and SACs of fibers with CF above the limit of pure-tone phaselocking have the same shape. Bin per bin subtraction of XACs from SACs results in "difcors," which reveal response components that change on inverting the polarity, and therefore reflect synchronization to the fine structure of the effective stimulus waveform.

\section{Results}

\section{Responses of TB fibers to broadband noise}

We report results for 340 TB fibers obtained from 16 cats and for 362 AN fibers obtained from 12 cats. We first illustrate responses from individual fibers, then show population data.

Figure 1 shows dot rasters of responses of a PHL fiber with a CF of $350 \mathrm{~Hz}$ (top row) and an AN fiber with a CF of $375 \mathrm{~Hz}$ (bottom row). The left dot rasters are obtained by repeatedly presenting a short pure tone at CF. The vertical alignment in the dot rasters indicates phase locking to the fine structure of the tone. When compared with the AN fiber (Fig. 1C), the alignment in the dot rasters of the PHL fiber (Fig. $1 A$ ) is better. This enhanced phase locking can be quantified with the vector strength metric $R$, which equals 0.98 for the PHL fiber and 0.76 for the AN fiber.

The dot rasters on the right (Fig. $1 B, D$ ) show responses to repeated frozen broadband noise. Here, the vertically aligned dots are irregularly spaced as expected for responses to an aperiodic stimulus. The scatter around preferred timings is, on average, larger in the AN fiber responses (Fig. 1D) than in the PHL fiber responses (Fig. $1 B$ ), as was also the case in the pure tone responses. Moreover, there is higher consistency in the response of the PHL fiber compared with the AN fiber, in the sense that the spike pattern from trial to trial shows less "skipped events" in the PHL than in the AN fiber. The $\mathrm{CR}_{\max }$ and CI calculated from the SACs of the noise responses (see Materials and Methods) are 22.5 coincidences/s and 7.5 for the PHL fiber versus 1.8 coincidences/s and 5.4 for the AN fiber responses. Thus, the PHL fiber shows 7.5 times as many coincidences as expected based on chance (5.4 times as many for the AN fiber). Note that the contrast in $\mathrm{CR}_{\max }$ values is larger than that in the CI values: we will return to this observation below.

Figure 2 shows dot rasters of responses to broadband noise from a $\mathrm{PL}_{\mathrm{N}}$ fiber with a CF of $7600 \mathrm{~Hz}$ (top row) and an $\mathrm{AN}$ fiber with a CF of $7300 \mathrm{~Hz}$ (bottom row). Again, spikes tend to align vertically, showing a consistent response across repetitions of the noise token, but here the patterning reflects envelope time locking, as will be further illustrated below. Compared with Figure 1, the vertical alignment is less clear and is barely visible for the high-CF AN fiber (Fig. 2, bottom row). The $\mathrm{CR}_{\max }$ and $\mathrm{CI}$ values are correspondingly low but still higher for the TB than for the AN fiber (1.6 coincidences/s and 2.3 for the $\mathrm{PL}_{\mathrm{N}}$ fiber vs 0.6

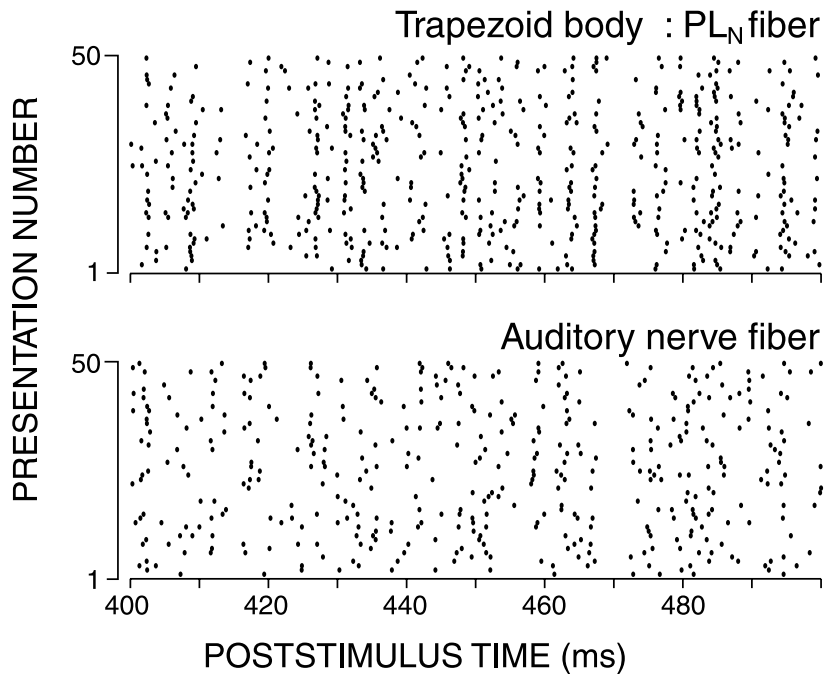

Figure 2. Dot rasters of broadband noise responses from a $\mathrm{PL}_{N}$ (top row) and an $\mathrm{AN}$ (bottom row) fiber. (Fs and SR were $7600 \mathrm{~Hz}$ and 27 spikes/s for the $P L_{N}$ fiber and $7300 \mathrm{~Hz}$ and 10 spikes $/ \mathrm{s}$ for the AN fiber. The sound level was $50 \mathrm{~dB}$ SPL in both cases.

coincidences/s and 1.7 for the AN fiber). The difference between $\mathrm{TB}$ and $\mathrm{AN}$ fibers is easy to visualize with dot rasters, but these displays are not amenable to a quantitative description of temporal behavior. In the next section, we use SACs for that purpose.

\section{SACs of responses to broadband noise}

Figure 3 shows typical SACs of four AN fibers (left column) and $12 \mathrm{~TB}$ fibers (columns 2-4) of low-CF $(<1 \mathrm{kHz}$, first row), mid-CF (second row), and high-CF ( $>3 \mathrm{kHz}$, third and fourth row). All SACs are normalized and therefore asymptote toward unity at long delays.

The first row shows normalized SACs of one low-CF AN fiber (Fig. 3A), two PHL fibers (Fig. 3B,C), and one chopper fiber (Fig. $3 D)$. Their basic shapes are similar and show a damped oscillation whose frequency reflects the CF of the fibers. However, compared with the AN fiber (Fig. $3 A$ ) with similar CF, the PHL fibers of Figure 3, $B$ and $C$, have higher and narrower peaks at delay zero. The CI of the fiber in Figure $3 C$ is 14.9 , which is $\sim 50 \%$ higher than ever obtained in AN fibers with similar CF (Louage et al., 2004). The SAC of the low-CF chopper unit (Fig. 3D) differs from AN not so much in its CI, but in the shallowness of the oscillation, which is superimposed on a broad peak. This combined shape was never seen with AN fibers of similar CF (Louage et al., 2004).

The second row of Figure 3 shows SACs for CFs of $\sim 2 \mathrm{kHz}$. In this frequency region, $\mathrm{SACs}$ of $\mathrm{PL}$ and $\mathrm{PL}_{\mathrm{N}}$ fibers resemble those of AN fibers. In contrast, the SAC of the chopper fiber (Fig. $3 H$ ) differs markedly: it does not oscillate. Rather, its shape is a single broad central peak.

The third and fourth row of Figure 3 shows SACs of high-CF fibers. All these SACs show a central peak, to a variable extent surrounded by a shallow trough. The SACs of the PL fibers (Fig. $3 J, N)$ are similar to those in the AN, but the SACs of the $\mathrm{PL}_{\mathrm{N}}$ fibers (Fig. $3 K, O$ ) have much higher central peaks and more pronounced troughs than AN fibers (Fig. 3I,M), which is also the case for the two chopper fibers (Fig. $3 L, P$ ).

As shown previously for AN fibers (Joris, 2003; Louage et al., 2004) and further illustrated below, the features in SACs of high-CF fibers reflect envelope time-locking. The envelope of relevance here is not that of the acoustic stimulus but the enve- 

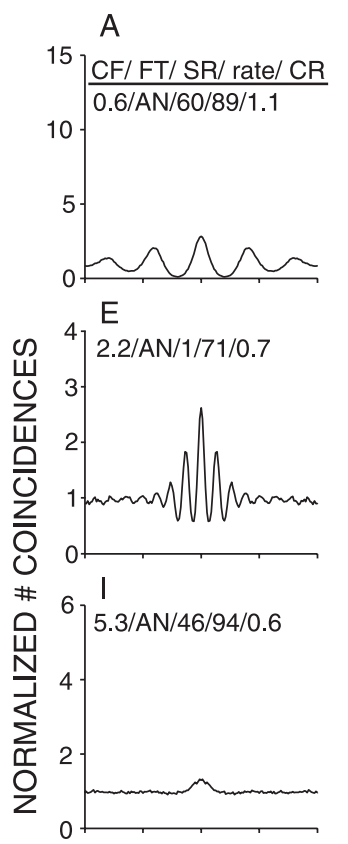

$\mathrm{M}$

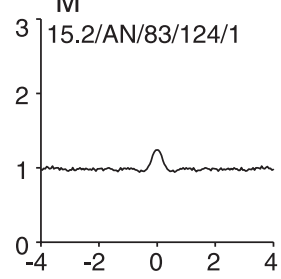

\section{B}
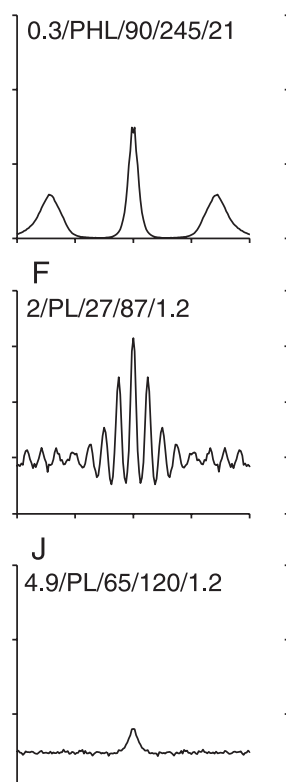

$\mathrm{N}$

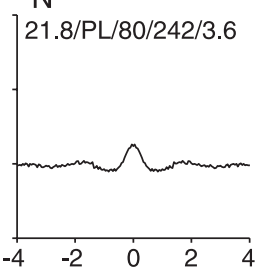

$\operatorname{DELAY}(\mathrm{ms})$
C

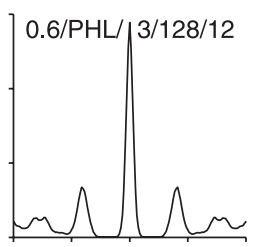

$\mathrm{G}$

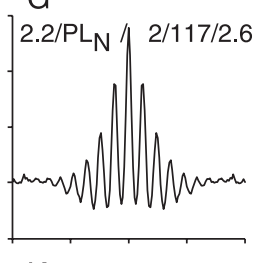

$\mathrm{K}$
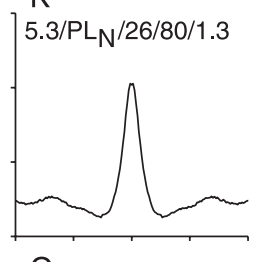

O
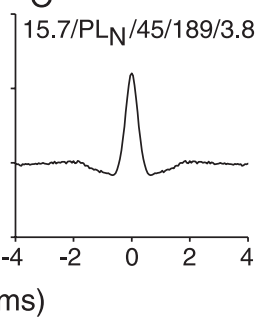

$\mathrm{D}$

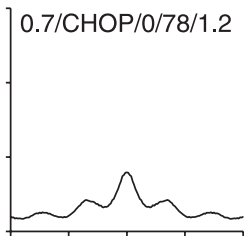

$\mathrm{H}$
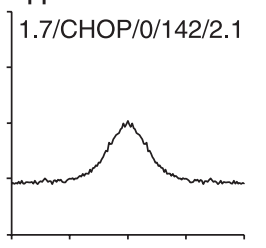

$\mathrm{L}$
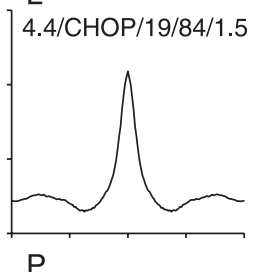

9.1/CHOP/21/275/5.3

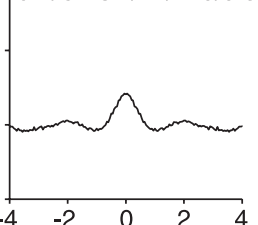

Figure 3. Examples of $S A C s$ of responses to broadband noise from four AN fibers (left column) and 12 TB fibers. All abscissas have the same scale. For each row, ordinate scales in the left column apply to all other columns. In all cases, the stimulus level was $\sim 50 \mathrm{~dB}$ SPL. The CF, fiber type, SR, average firing rate (in spikes $/ \mathrm{s}$ ), and $\mathrm{CR}_{\max }$ (coincidences/s) are indicated in the left top corner of each panel.

A $\mathrm{CF}(\mathrm{Hz}) / \mathrm{FT} / \mathrm{SR}(\mathrm{sp} / \mathrm{s}) \quad \mathrm{B}$

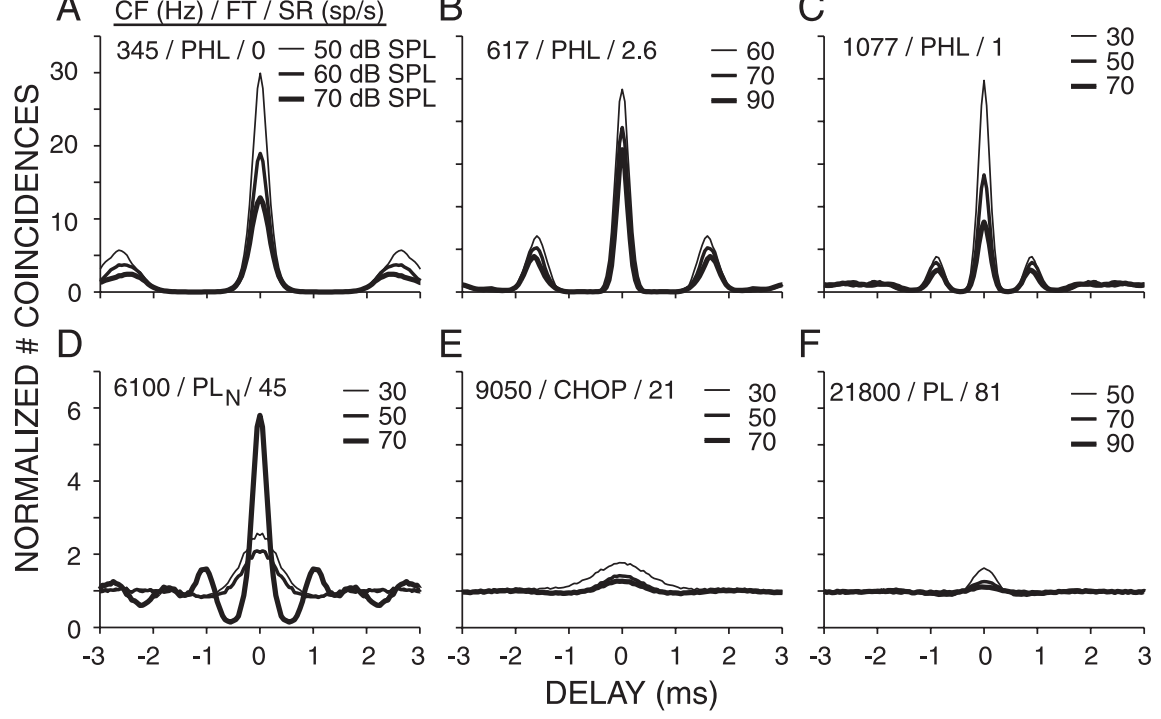

Figure 4. Effects of SPL on normalized SACs obtained from responses to broadband noise of six TB fibers. Top row shows low-CF, and the bottom row shows high-CF fibers. The abscissas of all panels are on the same scale. For each row, the ordinate scale in the left column applies to all other columns, but note that it differs between the top and bottom rows. CF, fiber type, SR, and SPL are indicated in each panel. lope of the "effective stimulus" i.e., the bandpass-filtered version of the stimulus that appears at the inner hair cell or cells from which the AN or TB fibers ultimately derive their input. Some SACs of high-CF neurons (Fig. $3 K, L, N-P$ ) tend to oscillate, but at a frequency much lower than their $\mathrm{CF}$. We surmise that this response periodicity reflects tuning to envelope frequencies, a point to which we will return in the Discussion.

In summary, TB responses often show enhanced temporal features in their response to noise and a different weighting of fine structure versus envelope, compared with AN fibers. The feature that we will focus on most is the higher peak height or CI. Because phase locking to pure tones and amplitude-modulated tones is known to be SPL-dependent, we first examine how that stimulus parameter affects SACs.

\section{Effect of SPL on normalized SACs}

In some fibers, noise responses were obtained at several sound levels. Figure 4 illustrates effects of SPL on normalized SACs of three low-CF (top row) and three high-CF (bottom row) TB fibers. In the absence of sound, normalized SACs are straight lines at unity. At suprathreshold levels, SPL could affect the height (Fig. $4 A-F$ ) and the width (Fig. $4 E$ ) of the central peak, the oscillation frequency of the SAC (Fig. 4A-D), and the damping of the SAC (Fig. 4C, where the ratio of central to secondary peaks changes with SPL), but in the vast majority of fibers the basic shape of the SAC was unaltered. However, in a few fibers the phenomenon illustrated in Figure $4 D$ was observed. The SACs of this high-CF $\mathrm{PL}_{\mathrm{N}}$ fiber are single-peaked for the two lowest SPLs, as expected from the synchronization to the envelope (Fig. 3). The SAC at $70 \mathrm{~dB}$, however, is oscillatory. It is important that the frequency of this oscillation $(\sim 700 \mathrm{~Hz})$ is well below 6.1 $\mathrm{kHz}$, the $\mathrm{CF}$ of the fiber. The XAC between the noise and its polarity-inverted version (data not shown) oscillated in antiphase, showing that the oscillations reflect fine structure rather than envelope coding. At $70 \mathrm{~dB}$, therefore, the fiber behaves much like a low-CF fiber (Fig. 4C). Such a dramatic downshift of the region of spectral dominance was observed for seven of nine $\mathrm{PL}_{\mathrm{N}}$ fibers with CFs between 4 and $7 \mathrm{kHz}$. We did not observe this phenomenon in AN fibers, chopper fibers, or PL fibers.

We now turn to effects of SPL on the height of the central peaks (the CI) (Fig. $5 A, B)$ and their width at halfheight (halfwidth) (Fig. 5C,D). With increasing SPL, $\mathrm{CI}$ increases from unity to a maximum 
A
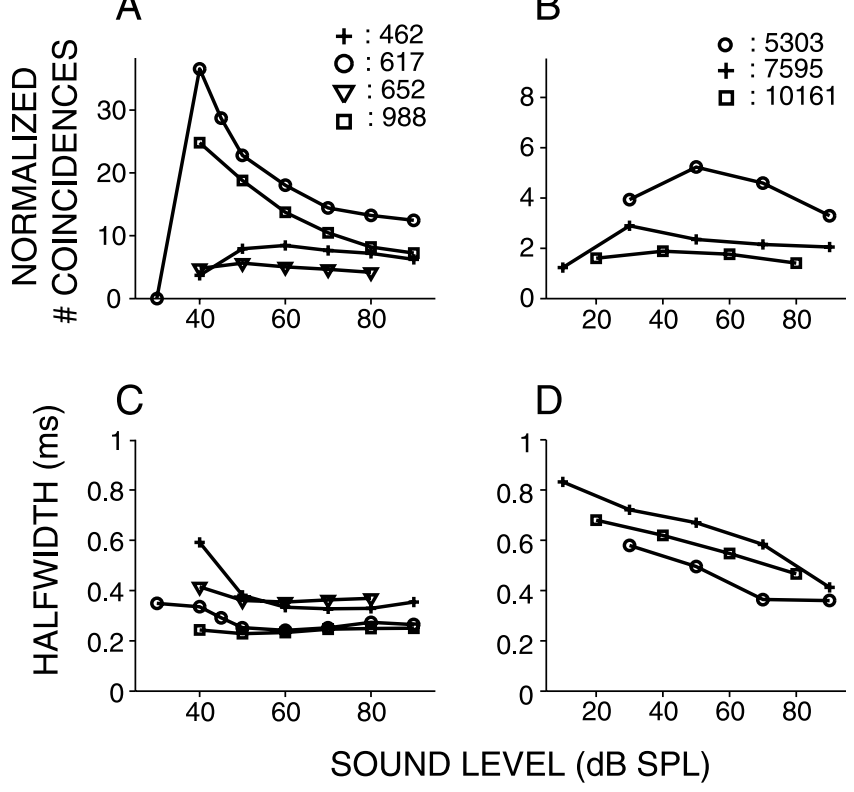

Figure 5. Influence of SPL on correlation index (top row) and halfwidth (bottom row) of the central peak of SACs, for low-CF PHL fibers (left column) and high-CF PL fibers (right column). Each line represents a single fiber. In each column, the corresponding symbols of the top and bottom rows represent the same fiber. CFs (in Hertz) are indicated in the top right corners of the top panels.

value at intermediate SPLs $(30-60 \mathrm{~dB})$ and decreases with further increases of SPL. This is true for both low-CF fibers (Fig. $5 A$ ) and high-CF fibers (Fig. 5B), and generally parallels the effects seen in AN fibers. An important difference between TB and AN, however, is the behavior of high-CF fibers at high intensities. In most AN fibers, CI drops to values $<1.5$, revealing very poor envelope coding at high intensities. In the TB, on the other hand, the CI at high sound levels is typically larger (Fig. 5B).

Halfwidth of the central peak quantifies the temporal precision of the coupling of spikes to the stimulus (Louage et al., 2004). In low-CF PHL fibers, halfwidth is largest around threshold, narrows toward a minimum at intermediate SPLs, and slightly broadens at high SPLs (Fig. $5 C$ ). This pattern of SPL dependency is similar to what is observed with low-CF AN fibers (Louage et al., 2004). In high-CF TB fibers, halfwidth is largest around threshold and decreases monotonically with increasing SPL (Fig. $5 D$ ) by almost a factor 2 over a range of $\sim 60 \mathrm{~dB}$. Overall, halfwidths are larger for these high-CF fibers than for the low-CF fibers shown in Figure $5 C$ (see Figs. 7, 12A). This combination of SACs in high-CF fibers becoming lower (Fig. $5 B$ ) and narrower (Fig. 5D) at high SPLs was also observed in the AN (Louage et al., 2004) (see Fig. 15) and reflects two somewhat counterintuitive trends in the spike timing of these fibers. On the one hand, increase in SPL generates relatively fewer spikes that are consistently timed across presentations; on the other hand, the spikes of this reduced subset are timed more accurately. The first trend agrees with the loss of phase locking to the envelope with high sound levels (Smith and Brachman, 1980; Joris and Yin, 1992; Wang and Sachs, 1993), but an interpretation of both trends combined will require a detailed description of the mechanisms of spike generation.

\section{Dependence of coincidence rate, $\mathrm{CI}$, and average rate on $\mathrm{CF}$} Figure $6 A$ shows the $\mathrm{CR}_{\max }$ from all $\mathrm{TB}$ fibers. Multiple points connected by a vertical line represent those fibers from which we obtained SACs at more than one SPL. When only one SPL was tested, a single data point represents a single fiber. Different symbols represent the fiber type. The broadband noise was presented at levels ranging from 45 to $90 \mathrm{~dB}$, with a median of $70 \mathrm{~dB}$ SPL. The top limit of coincidence rate of AN fibers is shown with a solid line and had a maximum of $\sim 5.5$ coincidences $/ s$ for CFs of $\sim 400 \mathrm{~Hz}$, decreasing to values of $\sim 1.5$ coincidences/s for CFs $>1$ $\mathrm{kHz}$. Of the $340 \mathrm{~TB}$ fibers, 165 yielded a coincidence rate higher than the top limit encountered in AN fibers of similar CF. Higher coincidence rates of TB fiber responses occurred over the whole range of CFs, but were most obvious at $<1$ and $>6 \mathrm{kHz}$. Also, the incidence of a higher coincidence rate differed between fiber types: 21 of 28 for chopper fibers, 65 of 123 for $\mathrm{PL}_{\mathrm{N}}$ fibers, 35 of 68 PHL fibers, and 23 of 79 PL fibers had higher coincidence rates than ever observed in AN fibers of similar CF.

As discussed previously (Louage et al., 2004), the coincidence rate of a fiber depends on both its average firing rate and its tendency to fire spikes at the same temporal position after repeated stimulation with the same stimulus. Indeed, the more spikes available in two spike trains, the higher the probability of finding a spike with the same timing. To separate the relative contribution of these two aspects of the response to the data of Figure $6 A$, we plot the $C I$ in $B$ and the average rate in $C$. In the $A N$, CIs range from a value of 10 at the very lowest CFs to $\sim 1.8$ at CFs $>5 \mathrm{kHz}$ (Louage et al., 2004). Of 340 TB fibers, 114 had CIs higher than the top limit encountered in AN fibers of similar CF. Such "enhanced" CIs were most dramatic at low CFs but occurred throughout the entire CF range. Again, the incidence of such CIs differed between fiber types: in 61 of 123 for $\mathrm{PL}_{\mathrm{N}}$ fibers, 31 of 68 for PHL fibers, 5 of 79 for PL fibers, and 6 of 28 for chopper fibers. Thus, even when average rate effects are eliminated, differences between TB fibers and AN fibers persist.

Figure $6 C$ shows the average rate for the same fibers as in Figure $6 \mathrm{~A}$. For visual clarity only one value is presented: the maximum average rate obtained. Overall, TB fibers had higher maximal firing rates than AN fibers: the mean maximal rate was 91 spikes/s for AN fibers and 130 spikes/s for TB fibers (122 spikes/s for PL fibers, 124 spikes/s for PHL fibers, 124 spikes/s for $\mathrm{PL}_{\mathrm{N}}$ fibers, and 189 spikes/s for chopper fibers). TB fibers with CFs $>1 \mathrm{kHz}$ tended to have higher average rates with increasing CFs; we did not observe this trend for AN fibers.

In summary, Figure 6 shows that many TB fibers show higher $\mathrm{CR}_{\max }$ than AN fibers of comparable CF and that this can be attributed to improved time locking or to higher average firing rates. The second factor is particularly important at CFs $>5 \mathrm{kHz}$, whereas both factors contribute to the high CIs at CFs $<5 \mathrm{kHz}$.

\section{CF dependence of halfwidth}

In low-CF AN fibers, SACs show a clear CF dependence in their halfwidth (Louage et al., 2004). Figure 7 shows halfwidths for 72 low-CF TB fibers. Fibers with CFs $>1 \mathrm{kHz}$ are not included because such fibers synchronize mainly to the envelope of the effective stimulus. To visualize the datapoints with sufficient detail, the range of halfwidths is limited to $1 \mathrm{~ms}$, thereby clipping the data for two TB fibers. The solid lines outline the range of halfwidths in the AN, based on 203 SACs obtained from 99 AN fibers pooled from eight cats (Louage et al., 2004). In the AN, halfwidth tends to decrease with $\mathrm{CF}$, as expected from the concomitant increase in the DF (see Materials and Methods) (Fig. 3). A similar trend is present in the TB fibers but, importantly, for 38 of $69 \mathrm{~TB}$ fibers, either PHL, PL, $\mathrm{PL}_{\mathrm{N}}$, or no-PSTH, at least one SAC shows a halfwidth smaller than in AN fibers with similar CF. We have 


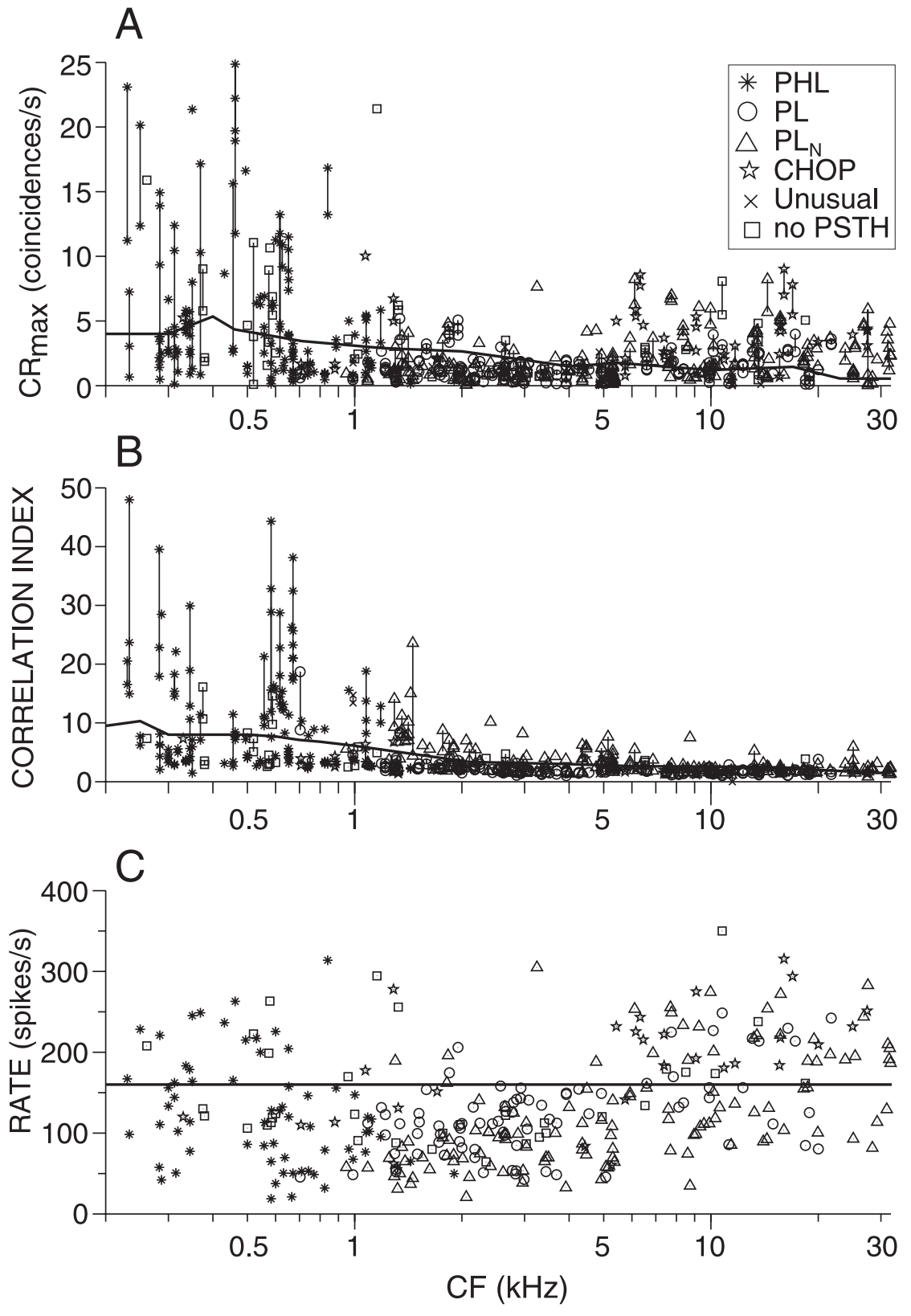

Figure 6. $\quad \mathrm{CR}_{\max }(A)$, correlation index $(B)$, and maximal average firing rate $(C)$ for responses to noise, plotted versus $C F$ for a population of $340 \mathrm{~TB}$ fibers pooled from 16 cats. Each vertical line connecting two or more data points represents data from a single fiber at multiple SPLs. The thick solid line in each panel indicates the top limit of values from a population of 360 AN fibers.

only three low-CF chopper fibers, but interestingly two of them had larger halfwidths than AN fibers.

\section{Dominant frequency of SACs}

Figure $8 A-C$ shows the Fourier spectrum of SACs of three TB fibers. The DF of each spectrum is indicated by an asterisk. Figure $8 \mathrm{D}$ shows the DF of $307 \mathrm{~TB}$ and 290 AN fibers plotted against their CF obtained from the threshold-tuning curve. Again, vertical lines connecting data points obtained at different SPLs are shown for TB data but are omitted for AN data. For AN fibers, the DF of SACs shows a good match to CF up to $\sim 3 \mathrm{kHz}$ and drops to much lower values for $\mathrm{CFs}>4 \mathrm{kHz}$, as expected from the pure tone phase-locking limit. Thus, in AN fibers, the stimulus feature (fine structure or envelope) that determines the DF of the SAC changes over a relatively narrow transition zone of CFs. Compared with AN fibers, this transition zone is smeared out in $\mathrm{TB}$ fibers over a broader range of CFs and is shifted toward lower CFs: it ranged from $\sim 1.4-2.5 \mathrm{kHz}$ for $\mathrm{PL}_{\mathrm{N}}$ fibers, and from 2 to $3.8 \mathrm{kHz}$ for PL fibers. The downshift of the transition zone is most prominent for chopper fibers, consistent with their lower pure-tone phase-locking limit (Bourk, 1976; Rhode and Smith, 1986; Blackburn and Sachs, 1989; Winter and Palmer, 1990; Joris et al., 1994a).

\section{Synchronization to fine structure}

The changing shape of SACs with CF (Fig. 3) reflects synchronization to different waveform features at different CF regions: fine structure versus envelope for low-CF and high-CF fibers, respectively. In the analysis so far, these two forms of synchronization were not addressed separately, and the data shown so far therefore reflect a mixture of the two forms of synchronization. Joris (2003) proposed a method to disambiguate synchronization to fine structure and envelope by constructing difcors from SACs and XACs (see Materials and Methods). Figure 9 illustrates SACs and XACs (rows 1 and 3; thin and thick lines, respectively) and difcors (rows 2 and 4) of two AN fibers (left column) and four TB fibers (middle and right columns). Difcors either oscillate at a frequency close to CF (Fig. 9D-F,J,K) or are a straight line at zero level, when responses are not synchronized to the fine structure (Fig. 9L). The central peaks of the difcors of the PHL fiber (Fig. 9E) and the PL fiber (Fig. 9K) are higher than those of the difcors of CF-matched AN fibers (Fig. 9D,J). The difcor peaks of the two chopper fibers, however, are lower than those of AN fibers (Fig. 9F,L). The responses of the chopper fiber with a CF of $1705 \mathrm{~Hz}$ (Fig. 9I) are, unlike those of the AN or PHL fibers with similar CF, totally insensitive to polarity changes of the fine structure and are thus completely envelope-based.

The relation between difcor peakheight and CF is illustrated for a large group of TB fibers in Figure 10. For comparison, the solid lines indicate the range of difcor peak heights of AN fibers (Louage et al., 2004), which reach a maximal value of 10 at the lowest CFs and vanish to values near 0 at $>4 \mathrm{kHz}$, reflecting the absence of phase locking to the fine structure of the effective stimulus at these CFs. The most striking observation is again the presence of higher difcor peak heights in TB fibers than ever seen in the AN, restricted to CFs in the phase locking range. Fibers from all PSTH categories contribute to such datapoints. Fibers with peak heights that are lower than ever seen in AN fibers of such CF consist predominantly of choppers. Note that two $\mathrm{PL}_{\mathrm{N}}$ fibers with $\mathrm{CF}$ between 3 and $5 \mathrm{kHz}$ have difcor peak heights exceeding the range of the AN (Fig. 10, arrows). We will return to these fibers below. 


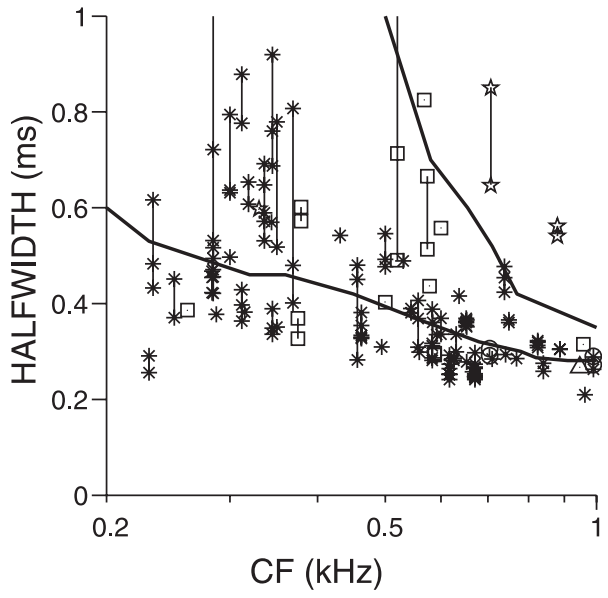

Figure 7. CF dependence of halfwidth of SACs to noise obtained from a population of low-CF TB and AN fibers. Data from single fibers at multiple SPLs are connected by a vertical line. The thick solid lines indicate the range of halfwidth values obtained from 99 AN fibers. Symbols are the same as those shown for Figure 6 .
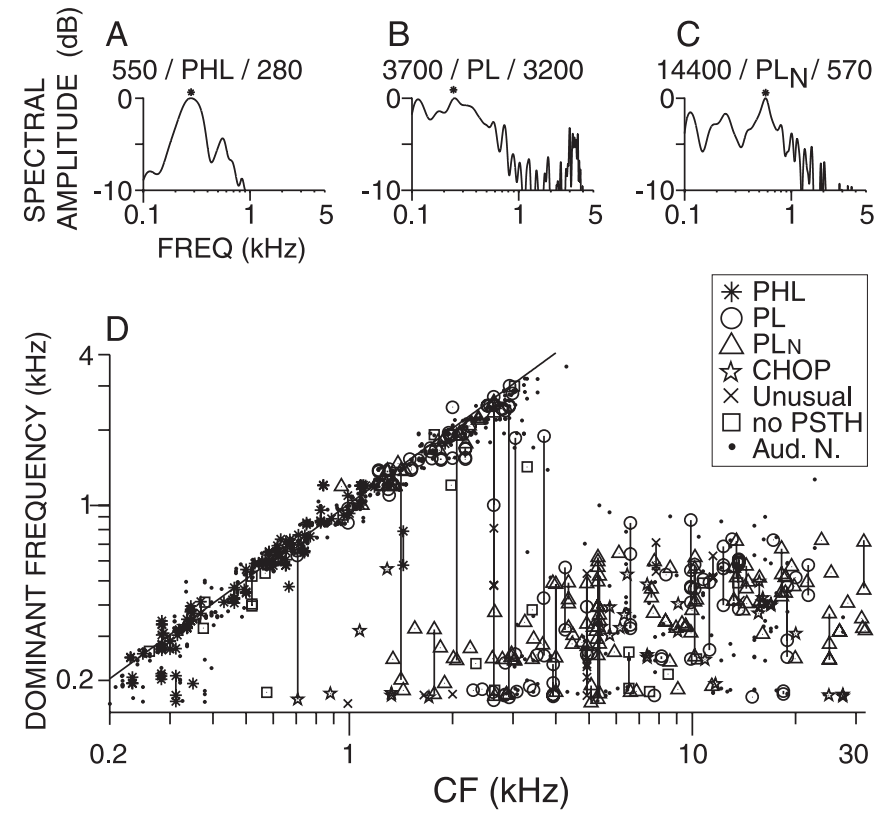

Figure 8. $A-C$, Amplitude spectra $\left(10 \cdot \log _{10}\right)$ of $S A C S$ of a low-, mid-, and high-CF TB fiber. The asterisk indicates the DF or spectral maximum. D, DF of SACs versus CF. The oblique solid line graphs equality. Each vertical line connects data from single fibers at multiple SPLS.

\section{Synchronization to envelope}

The transition region over which temporal coding changes from being dominated by fine structure to envelope occurs at lower CFs in binaural cells in the inferior colliculus than in the AN, and it has been proposed that part of this shift may already occur in cochlear nucleus bushy cells (Joris, 2003). The relative contribution of fine structure and envelope can be quantified by calculating the ratio of the amplitude of peak and trough of SAC and XAC, respectively, at zero delay (Louage et al., 2004) (Fig. 9). The higher this ratio, the less the response is sensitive to stimulus polarity and the more it is thus envelope-dominated. Figure 11 illustrates that this ratio shows a sigmoidal relationship with CF in TB fibers. Indeed, compared with the AN (solid lines) the relationship is shifted to the left along the abscissa for the various subpopulations of fibers, particularly so for the choppers. A downward shift is also observed with the DF (Fig. 8D) and in phase locking to pure tones (Joris et al., 1994a). Note that the $\mathrm{XAC} / \mathrm{SAC}$ ratio of three high-CF $\mathrm{PL}_{\mathrm{N}}$ fibers is smaller than that of AN fibers of similar CF (Fig. 11, arrows). Closer inspection revealed that these responses are all obtained at high SPLs and that they all showed the large downshift of spectral dominance explained earlier in the discussion of Figure $4 D$.

The dotted line in Figure 11 illustrates our criterion for envelope-dominated responses. We define responses with the $\mathrm{XAC} / \mathrm{SAC}$ ratio $>0.9$ as envelope-dominated. This subset is further analyzed in Figure 12. Figure $12 \mathrm{~A}$ shows the halfwidth of these fibers as a function of CF. The two curves in this plot indicate the range of halfwidths of high-CF AN fibers stimulated at 50 dB SPL; these curves are extracted from Louage et al. (2004), their Figure 17. The symbols in Figure $12 \mathrm{~A}$ represent halfwidths of various types of TB fibers for noise levels of 40-60 dB SPL. In both AN and TB fibers, halfwidths decrease with increasing CF to reach minimum values of $\sim 400 \mu \mathrm{s}$. TB fibers had similar or larger halfwidths compared with AN fibers. Larger halfwidths occurred most frequently with chopper fibers (asterisks).

For comparison with data in inferior colliculus (see Discussion), Figure $12 \mathrm{~B}$ shows the modulation depth of the same envelope-dominated responses as Figure $12 \mathrm{~A}$. The modulation depth, defined as (maximum -minimum)/(maximum) of the SAC, quantifies the degree to which the SAC is modulated by changes in delay. The horizontal dotted lines in Figure $12 \mathrm{~B}$ indicate the limits of modulation depths of SACs obtained from AN responses; these limits are extracted from the study by Joris (2003), his Fig. 4. Figure $12 B$ shows that SACs of TB responses are more deeply modulated that SACs of AN responses. This contrast with AN fibers is most pronounced for $\mathrm{PL}_{\mathrm{N}}$ fibers.

\section{Comparison of pure tone responses with noise responses}

An important issue is whether those TB fibers that show enhanced synchronization to short tone bursts at CF also show enhanced synchronization to sustained broadband noise. Figure 13 illustrates the relation between vector strength $R$ in response to short $\mathrm{CF}$ tones on the one hand, and the two types of correlation metrics, $\mathrm{CI}$ and $\mathrm{CR}_{\max }$, in response to noise bursts on the other hand. We restrict our analysis to $\mathrm{CFs}<800 \mathrm{~Hz}$, where enhanced synchronization to pure tones is most marked (Joris et al., 1994a).

Figure $13 A$ shows the relation between the CI obtained from responses to broadband noise (ordinate), and $R$ obtained from responses to short tone bursts at CF (abscissa). Each data point represents the two metrics obtained from the same fiber; for fibers tested at multiple SPLs, only the highest values of $R$ and CI are shown. Clearly, higher $R$ is associated with a higher CI, but there is a considerable range of CI values in "high-sync" fibers $(R>0.9)$ (Joris et al., 1994a). This range is to some extent related to spontaneous rate (Fig. 13B): hi-sync fibers always have larger CI values than AN fibers of comparable SR, and low-SR TB fibers have generally higher CI values than high-SR TB fibers. The inverse correlation between SR and CI was also observed in AN fibers (Louage et al., 2004).

Figure $13 C$ shows the relation between $\mathrm{CR}_{\max }$ obtained from responses to broadband noise and $R$ obtained from responses to short tone bursts at CF. For each fiber, the maximum values of $R$ and $\mathrm{CR}_{\max }$ are shown if responses at multiple SPLs are available. Most PHL fibers (asterisks) have $\mathrm{CR}_{\max }$ values higher than $\mathrm{AN}$ fibers; high-sync fibers (encircled asterisks) reach the highest values and can have either low or high SR.

Figure 14 shows the halfwidths of SACs calculated from responses to broadband noise for PHL fibers. For each fiber, the smallest halfwidth is shown if responses at multiple SPLs are 


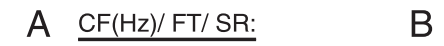

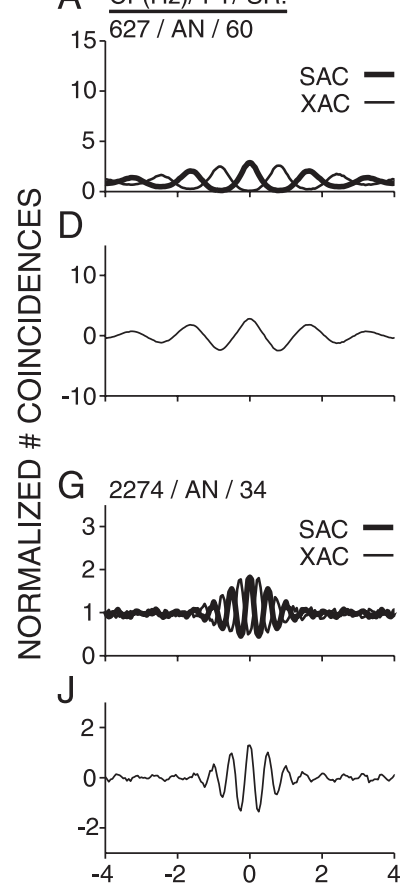

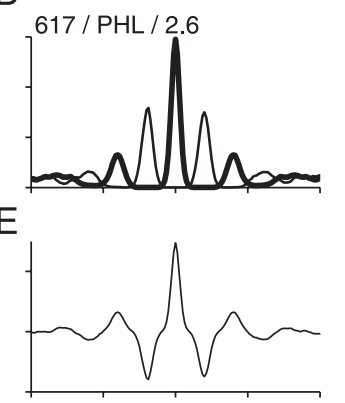
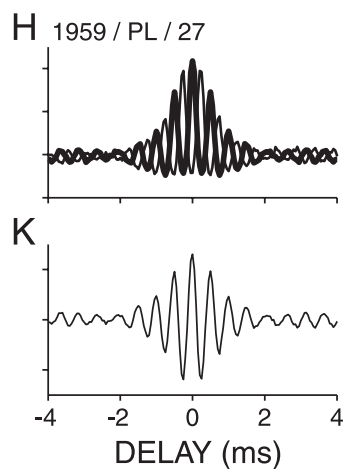

C
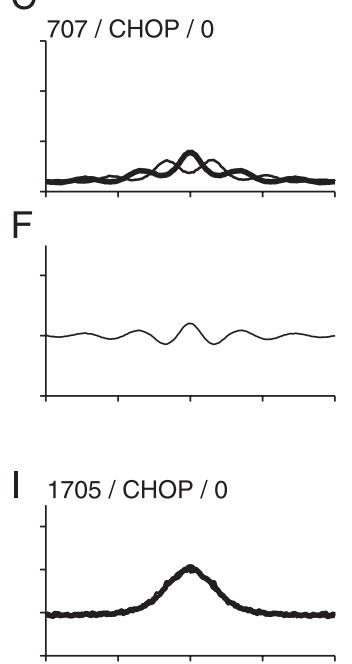

$\mathrm{L}$

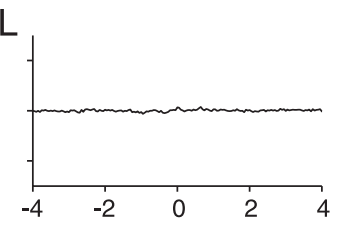

Figure 9. $A-C, G-I$, Examples of $S A C S$ (thick lines) and normalized XACS (thin lines) for two AN fibers (left column) and four TB fibers (middle and right column). $D-F$, $J-L$, Difcors obtained by subtraction of XACs from $S A C s$. The abscissas of all panels are on the same scale. Ordinate scales in the left column apply to all other columns, but differ between rows. The stimulus level for all fibers was $\sim 50 \mathrm{~dB}$ SPL. CF, fiber type, and SR are as indicated.

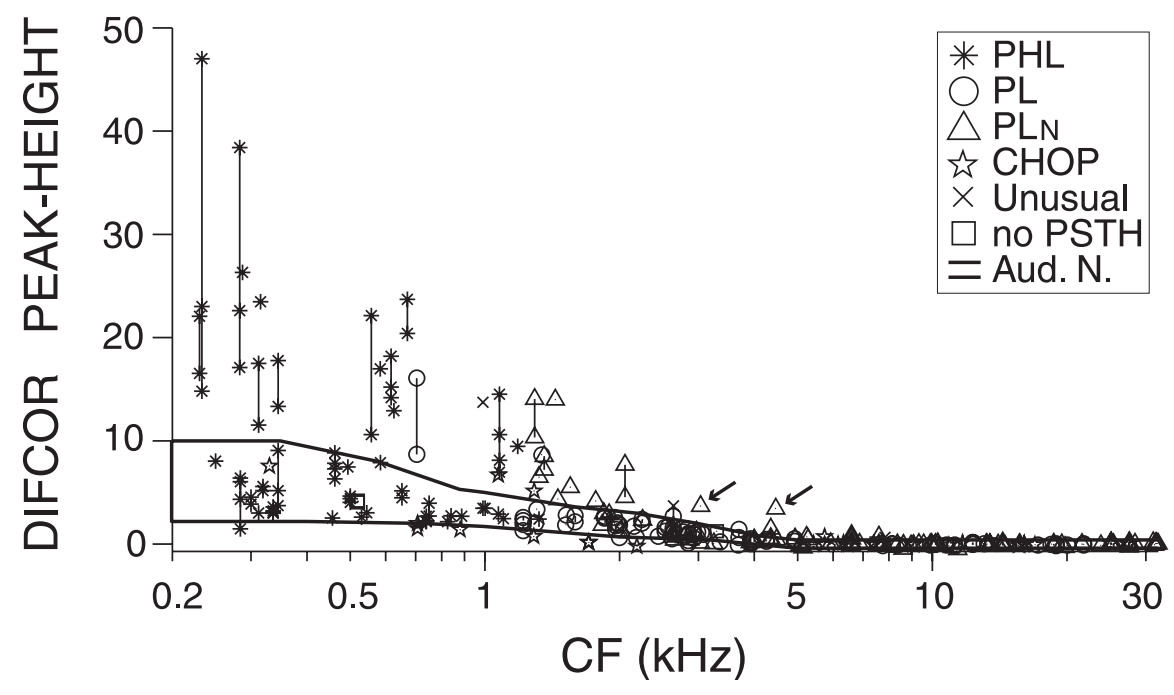

Figure 10. CF dependence of difcor peak heights for TB and AN fibers. The broadband noise was presented at $50-90 \mathrm{~dB}$ SPL for both TB and AN fibers. The thick solid lines illustrate the range of values for a population of AN fibers.

available. Fibers that had high-sync responses to pure tones at $\mathrm{CF}$ are marked by a circle. Most high-sync fibers have halfwidths smaller than those obtained from AN fibers, as indicated by the two solid lines.

\section{Discussion}

We obtained responses to broadband noise from the output fibers of the AVCN, quantified their temporal properties responses with autocorrelograms, and found enhanced temporal properties relative to AN fibers.

\section{Consistency and precision of firing}

We calculated normalized SACs and interpreted their peak value or CI as a metric for the consistency or reproducibility of the spike trains generated by a single neuron across repeated presentations of an identical stimulus (Louage et al., 2004). For example, a CI of 10 means that the responses of a neuron yield 10 times more spikes in the same temporal position than expected from chance. SACs of many low-CF TB fibers yielded not only higher CIs than AN fibers (Fig. 6) but also smaller halfwidths (Fig. 7), indicating better temporal precision. Taken together, these results can be interpreted as a reduction in internal noise of the neural response of AVCN neurons relative to the AN (Kiang, 1990). Our analysis also highlights that the generally larger average firing rate in AVCN neurons, compared with AN fibers, can also be an important contributor to an increased number of coincidences (Fig. 6). In retrospect this may be unsurprising, but nevertheless this difference between $\mathrm{AN}$ and AVCN has not been considered in discussions and models of binaural processing.

Other metrics for the measurement of temporal coding in the response to nonperiodic stimuli have been proposed (Strong et al., 1998; Szucs et al., 2004). Our choice of an autocorrelation- or coincidencebased analysis is motivated in our previous papers: it is a natural choice given the importance of the concepts of correlation and coincidence detection in binaural hearing (Colburn, 1996).

\section{Comparison with responses to pure tones}

Joris et al. (1994a) studied TB fibers with pure tones at CF and found that the majority showed higher $R$ values than AN fibers did, up to CFs of $1 \mathrm{kHz}$. At higher CFs, particularly $>2 \mathrm{kHz}$, the $R$ values of TB fibers tended to be lower than in the AN, although these fibers typically still showed enhanced synchronization to tones presented in their low-frequency tail (Joris et al., 1994b). The enhanced temporal encoding to broadband noise described here is also predominantly, although not exclusively, found at low CFs (Fig. 6). Enhanced encoding of fine structure is restricted to fibers in the phaselocking range (Fig. 10); the enhanced encoding at higher CFs reflects envelope coding and is discussed below.

The population distribution of enhanced encoding of noise differs in some respects from that of tones. Whereas nearly all fibers with $\mathrm{CF}<500 \mathrm{~Hz}$ are high-sync to tones (Joris et al., 1994b), the autocorrelograms to noise at these CFs are often within the AN limits (Figs. 6, 10). Thus, the degree of phase locking to pure tones is not entirely predictive of that to noise, 
and indeed a direct comparison shows that some fibers that are high-sync to tones are within the AN boundaries for the encoding of noise (Fig. 13A,C). However, when $\mathrm{SR}$ is taken into account (Fig. 13B,D), TB fibers are almost always near or above the top range of noise encoding in the AN. Our previous AN study (Louage et al., 2004) also revealed large differences in temporal encoding to noise between the different SR populations. Thus, more so than for pure-tone phase locking, SR is an important fiber property to consider when studying temporal aspects of responses to broadband stimuli.

A second difference between the population distributions to noise and tones is that the inversion (AN better phase-locked than TB) at CFs $>2 \mathrm{kHz}$ observed by Joris et al. (1994a) is not observed in the responses to noise. Nevertheless, the lower cutoff in the phase locking to tones in TB fibers is also present in the data to noise (Fig. 11). The temporal patterning of responses is dominated by fine structure at low CFs and by envelope at high CFs, and the transition region between these two regions is shifted to lower CFs in the TB relative to the AN. Using a different metric, Joris (2003) showed that in neurons of the inferior colliculus the transition region of ITD-sensitivity based on fine structure versus envelope was also shifted toward lower CFs, relative to the $\mathrm{AN}$, and proposed that this downshift could be partly explained by the reduced top limit of phase locking in TB fibers, which we here show is indeed present to noise (Fig. 11).

As expected, the temporal patterning of responses of low-CF fibers was dominated by frequencies close to $\mathrm{CF}$, yielding an excellent match between CF and DF (Fig. 8). However, in some $\mathrm{PL}_{\mathrm{N}}$ fibers the response was dominated by frequencies far below $\mathrm{CF}$, particularly at high SPLs (Figs. $4 D, 10,11$ ). A similar nonlinearity has been observed by Rhode and Smith (1986) and Godfrey et al. (1975) in onset fibers and by Joris et al. (1994b) in $\mathrm{PL}_{\mathrm{N}}$ fibers that showed entrainment when stimulated with a $500 \mathrm{~Hz}$ tone. Blackburn and Sachs (1990) showed that, at high SPLs, some $\mathrm{PL}_{\mathrm{N}}$ fibers with CFs up to $5 \mathrm{kHz}$ have higher driven rates to the vowel $/ \epsilon /$ than to $\mathrm{CF}$ tones and synchronize preferably to harmonics below $\mathrm{CF}$.

\section{Envelope synchronization}

Differences between the AN and TB in temporal encoding of noise also occur at high CFs, where TB fibers show higher peaks in their unnormalized SACs, larger modulation depths, and shapes that differ from the single-peaked SACs typically seen in the AN (Figs. 2, 3, 6, 12). Such differences are to be expected because temporal encoding at high CFs reflects envelope coding, which is known to differ between AN and AVCN (for review, see Joris et al., 2004). AVCN neurons generally show higher gain to envelopes of amplitude-modulated (AM) tones than AN fibers: this is probably related to the higher peaks in the SACs (Fig. 6). AVCN neurons also differ substantially in the cutoff frequency at which encoding of the envelope is lost. Modulation transfer functions (MTFs) of PL and $\mathrm{PL}_{\mathrm{N}}$ cells are generally low-pass in shape with high cutoffs, whereas choppers show bandpass MTFs with lower cutoffs (Frisina et al., 1990; Rhode and Greenberg, 1994; Joris and Yin, 1998). The tendency toward bandpass MTFs is probably related to the occurrence of the slightly oscillatory SACs in
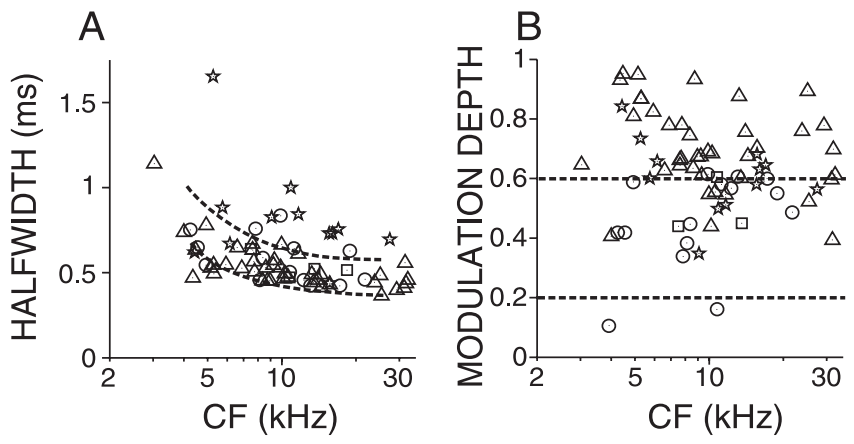

Figure 12. Halfwidths $(A)$ and modulation depths $(B)$ of SACs from envelope-dominated responses obtained at $40-60 \mathrm{dBSPL}$. The dashed lines indicate the extreme values obtained for AN fibers.

high-CF AVCN neurons (Fig. 3), because bandpass tuning in the modulation domain generates spike intervals at the preferred modulation period (Kim et al., 1990). Halfwidths of chopper fibers, but not PL or $\mathrm{PL}_{\mathrm{N}}$ fibers, tend to be broader than those of AN fibers (Fig. 12): this is probably related to the lower MTF cutoffs and best modulation frequencies in choppers compared with AN fibers (Frisina et al., 1990; Rhode and Greenberg, 1994; Joris and Yin, 1998). Firmer conclusions could be drawn by collecting both AM and noise data from the same TB neurons, which we have not done here.

To enable comparison with data from ITD-sensitive inferior colliculus neurons (Joris, 2003), we also calculated modulation depth (Fig. 12B), which is another metric to express SAC peak height relative to the number of coincidences based on chance. Modulation depth was consistently higher in high-CF TB fibers than in the AN, which probably contributes to the large modulation depths seen in the inferior colliculus.

\section{Morphological basis and mechanisms of enhanced synchronization}

Intra-axonal labeling established that the TB axons displaying enhanced synchronization to pure tones, recorded with the procedures followed here, project to the main superior olivary nuclei and thus derive from spherical or globular bushy cells (Smith et al., 1991, 1993; Joris et al., 1994a). Computational models (Roth- 
A
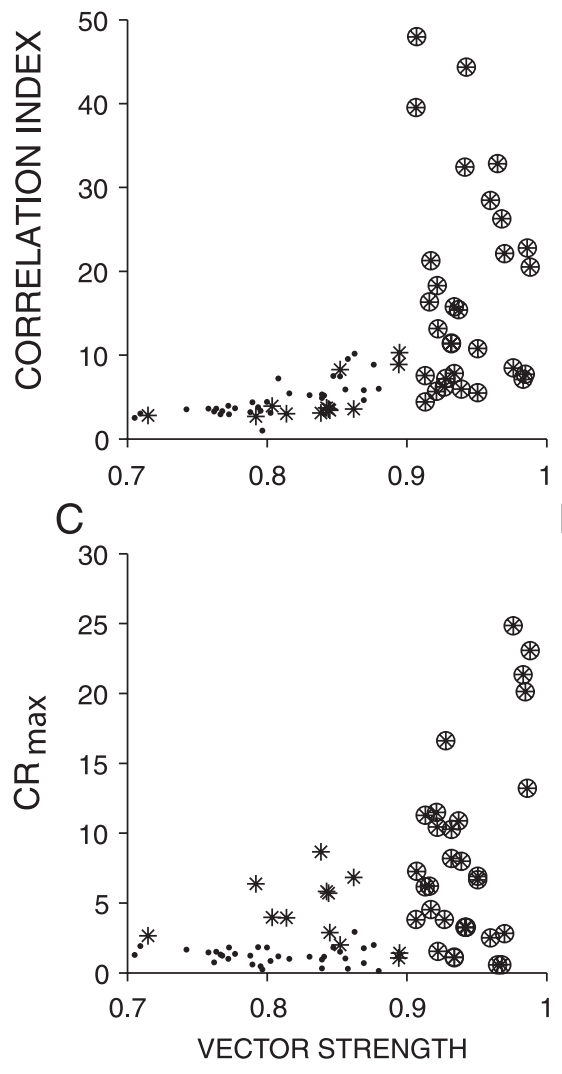

Figure 13. $\quad A, \mathrm{Cl}$ obtained from responses to broadband noise versus vector strength $(R)$ obtained from responses to short tone bursts at $C F$. When responses were obtained at more than one SPL, we chose the maximum $\mathrm{Cl}$ and $R$. Each data point represents the two metrics from a single AN or TB fiber. $B$, Cl from responses to broadband noise versus $S R . C, C R_{\max }$ obtained from the same responses as in $A . D, C R_{\max }$ from responses to broadband noise versus $S R$. Each asterisk represents a PHL fiber for which responses to $\mathrm{CF}$ tones were available. When the tonal responses yielded a vector strength $(R)$ of $>0.9$, the asterisk is encircled.

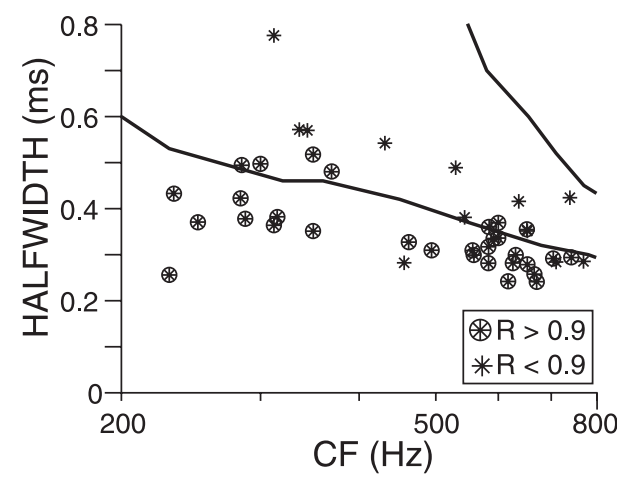

Figure 14. Halfwidth of SACs obtained from responses of PHL fibers to broadband noise. The solid lines indicate the range of values of halfwidths obtained from $99 \mathrm{AN}$ fibers.

man et al., 1993; Joris et al., 1994a; Rothman and Young, 1996; Kuhlmann et al., 2002; Ito and Akagi, 2005; Maki and Akagi, 2005) have achieved synchronization enhancement and entrainment by a process of monaural coincidence detection operating with a large number of subthreshold inputs. This applies to globular bushy cells, but the AN inputs to spherical bushy cells are both small in number and powerful, in the form of endbulbs of Held (Lorente de Nó, 1981; Ryugo and Sento, 1991), and it re- mains unclear how synchronization enhancement is achieved in the latter cells. Our data do not provide new insights in this issue, although they provide further constraints for computational models. Many of the cells that generated enhanced temporal encoding of tones or noise had high SR (Fig. 13B) and were located at depths corresponding to the dorsal component of the TB (data not shown): features that are both associated with spherical bushy cells (Brownell, 1975; Spirou et al., 1990; Smith et al., 1993). On the other hand, as mentioned, not all low-CF TB axons showed improved encoding of noise relative to the AN (Fig. 6), so it remains possible that some low-CF bushy cell inputs to the medial superior olive are AN-like.

\section{Functional significance}

It has been suggested that the temporal enhancement between the AN and bushy cells may serve to enhance ITD sensitivity (Joris et al., 1994a). The results presented here are in line with that suggestion because they are based on the counting of coincidences, and because coincidence detection is a fundamental component of all binaural models. However, it remains to be shown directly that, within a framework of coincidence detection, TB responses are effectively superior to AN responses in simple binaural tasks.

\section{References}

Batra R, Kuwada S, Fitzpatrick DC (1997) Sensitivity to interaural temporal disparities of low- and high-frequency neurons in the superior olivary complex. I. Heterogeneity of responses. J Neurophysiol 78:1222-1236.

Blackburn CC, Sachs MB (1989) Classification of unit types in the anteroventral cochlear nucleus: PST histograms and regularity analysis. J Neurophysiol 62:1303-1329.

Blackburn CC, Sachs MB (1990) The representations of the steady-state vowel sound $/ \epsilon /$ in the discharge patterns of cat anteroventral cochlear nucleus neurons. J Neurophysiol 63:1191-1212.

Bourk TR (1976) Electrical responses of neural units in the anteroventral cochlear nucleus of the cat. Cambridge, MA: MIT.

Brownell WE (1975) Organization of the cat trapezoid body and the discharge characteristics of its fibers. Brain Res 94:413-433.

Brughera AR, Stutman ER, Carney LH, Colburn HS (1996) A model with excitation and inhibition for cells in the medial superior olive. Aud Neurosci 2:219-233.

Carney LH, Yin TCT (1988) Temporal coding of resonances by lowfrequency auditory nerve fibers: single-fiber responses and a population model. J Neurophysiol 60:1653-1677.

Colburn HS (1996) Computational models of binaural processing. In: Springer handbook on auditory research (Hawkins H, McMullen T, eds), pp 332-400. New York: Springer.

Finlayson PG, Caspary DM (1991) Low-frequency neurons in the lateral superior olive exhibit phase-sensitive binaural inhibition. J Neurophysiol 65:598-605.

Fitzpatrick DC, Kuwada S (2001) Tuning to interaural time differences across frequency. J Neurosci 21:4844-4851.

Fitzpatrick DC, Batra R, Stanford TR, Kuwada S (1997) A neuronal population code for sound localization. Nature 388:871-874.

Frisina RD, Smith RL, Chamberlain SC (1990) Encoding of amplitude modulation in the gerbil cochlear nucleus. I. A hierarchy of enhancement. Hear Res 44:99-122.

Galambos R, Davis H (1943) The responses of single auditory nerve fibers to acoustic stimulation. J Neurophysiol 6:39-58.

Godfrey DA, Kiang NYS, Norris BE (1975) Single unit activity in the posteroventral cochlear nucleus of the cat. J Comp Neurol 162:247-268.

Goldberg JM, Brown PB (1969) Response of binaural neurons of dog superior olivary complex to dichotic tonal stimuli: some physiological mechanisms of sound localization. J Neurophysiol 32:613-636.

Hancock KE, Delgutte B (2004) A physiologically based model of interaural time difference discrimination. J Neurosci 24:7110-7117.

Ito K, Akagi M (2005) Study on improving regularity of neural phaselocking in single neurons of AVCN via a computational model. In: Auditory signal processing: physiology, psychoacoustics, and models 
(Pressnitzer D, de Cheveigné A, McAdams S, Collet L, eds), pp 91-99. New York: Springer.

Johnson DH (1980) The relationship between spike rate and synchrony in responses of auditory-nerve fibers to single tones. J Acoust Soc Am 68:1115-1122.

Joris PX (2003) Interaural time sensitivity dominated by cochlea-induced envelope patterns. J Neurosci 23:6345-6350.

Joris PX, Yin TCT (1992) Responses to amplitude-modulated tones in the auditory nerve of the cat. J Acoust Soc Am 91:215-232.

Joris PX, Yin TCT (1995) Envelope coding in the lateral superior olive. I. Sensitivity to interaural time differences. J Neurophysiol 73:1043-1062.

Joris PX, Yin TCT (1998) Envelope coding in the lateral superior olive. III. Comparison with afferent pathways. J Neurophysiol 79:253-269.

Joris PX, Carney LH, Smith PH, Yin TCT (1994a) Enhancement of neural synchronization in the anteroventral cochlear nucleus. I. Responses to tones at the characteristic frequency. J Neurophysiol 71:1022-1036.

Joris PX, Smith PH, Yin TCT (1994b) Enhancement of neural synchronization in the anteroventral cochlear nucleus. II. Responses in the tuning curve tail. J Neurophysiol 71:1037-1051.

Joris PX, Schreiner CE, Rees A (2004) Neural processing of amplitudemodulated sounds. Physiol Rev 84:541-577.

Kiang NYS (1990) Curious oddments of auditory-nerve studies. Hear Res 49:1-16.

Kiang NYS, Watanabe T, Thomas EC, Clark LF (1965) Discharge patterns of single fibers in the cat's auditory nerve. Cambridge, MA: MIT.

Kim DO, Sirianni JG, Chang SO (1990) Responses of DCN-PVCN neurons and auditory nerve fibers in unanesthetized cats to $\mathrm{AM}$ and pure tones: Analysis with autocorrelation/power-spectrum. Hear Res 45:95-113.

Klump RG, Eady HR (1956) Some measurements of interaural time difference thresholds. J Acoust Soc Am 28:859-860.

Köppl C, Carr CE (2003) Computational diversity in the cochlear nucleus angularis of the barn owl. J Neurophysiol 89:2313-2329.

Kuhlmann L, Burkitt AN, Paolini A, Clark GM (2002) Summation of spatiotemporal input patterns in leaky integrate-and-fire neurons: application to neurons in the cochlear nucleus receiving converging auditory nerve fiber input. J Comput Neurosci 12:55-73.

Lorente de Nó R (1981) The primary acoustic nuclei. New York: Raven.

Louage DH, van der Heijden M, Joris PX (2004) Temporal properties of responses to broadband noise in the auditory nerve. J Neurophysiol 91:2051-2065.

Maki K, Akagi M (2005) A computational model of cochlear nucleus neurons. In: Auditory signal processing: physiology, psychoacoustics, and models (Pressnitzer D, de Cheveigné A, McAdams S, Collet L, eds), pp 84-90. New York: Springer.

Mardia KV, Jupp PE (2000) Directional statistics. Chichester: Wiley.

Marsalek P (2001) Neural code for sound localization at low frequencies. Neurocomputing 38:1443-1452.

Moushegian G, Rupert AL, Gidda JS (1975) Functional characteristics of superior olivary neurons to binaural stimuli. J Neurophysiol 1975:1037-1048.

Paolini AG, FitzGerald JV, Burkitt AN, Clark GM (2001) Temporal processing from the auditory nerve to the medial nucleus of the trapezoid body in the rat. Hear Res 159:101-116.

Pfeiffer RR (1966) Classification of response patterns of spike discharges for units in the cochlear nucleus: tone-burst stimulation. Exp Brain Res 1:220-235.

Rhode WS, Greenberg S (1994) Encoding of amplitude modulation in the cochlear nucleus of the cat. J Neurophysiol 71:1797-1825.

Rhode WS, Smith PH (1986) Encoding timing and intensity in the ventral cochlear nucleus of the cat. J Neurophysiol 56:261-286.

Rose JE, Gross NB, Geisler CD, Hind JE (1966) Some neural mechanisms in the inferior colliculus of the cat which may be relevant to localization of a sound source. J Neurophysiol 29:288-314.

Rose JE, Brugge JF, Anderson DJ, Hind JE (1967) Phase-locked response to low-frequency tones in single auditory nerve fibers of the squirrel monkey. J Neurophysiol 30:769-793.

Rothman JS, Young ED (1996) Enhancement of neural synchronization in computational models of ventral cochlear nucleus bushy cells. Aud Neurosci 2:47-62.

Rothman JS, Young ED, Manis PB (1993) Convergence of auditory nerve fibers onto bushy cells in the ventral cochlear nucleus: implications of a computational model. J Neurophysiol 70:2562-2583.

Ryugo DK, Sento S (1991) Synaptic connections of the auditory nerve in cats: relationship between endbulbs of held and spherical bushy cells. J Comp Neurol 305:35-48.

Shackleton TM, Skottun BC, Arnott RH, Palmer AR (2003) Interaural time difference discrimination thresholds for single neurons in the inferior colliculus of Guinea pigs. J Neurosci 23:716-724.

Smith PH, Joris PX, Carney LHC, Yin TCT (1991) Projections of physiologically characterized globular bushy cell axons from the cochlear nucleus of the cat. J Comp Neurol 304:387-407.

Smith PH, Joris PX, Yin TCT (1993) Projections of physiologically characterized spherical bushy cell axons from the cochlear nucleus of the cat: evidence for delay lines to the medial superior olive. J Comp Neurol 331:245-260.

Smith RL, Brachman ML (1980) Response modulation of auditory-nerve fibers by AM stimuli: effects of average intensity. Hear Res 2:123-133.

Spirou GA, Brownell WE, Zidanic M (1990) Recordings from cat trapezoid body and HRP labeling of globular bushy cell axons. J Neurophysiol 63:1169-1190.

Spitzer MW, Semple MN (1995) Neurons sensitive to interaural phase disparity in gerbil superior olive: diverse monaural and temporal response properties. J Neurophysiol 73:1668-1690.

Strong SP, Koberle R, de Ruyter van Steveninck RR, Bialek W (1998) Entropy and information in neural spike trains. Phys Rev Lett 80:197-200.

Szucs A, Vehovszky A, Molnar G, Pinto RD, Abarbanel HD (2004) Reliability and precision of neural spike timing: simulation of spectrally broadband synaptic inputs. Neuroscience 126:1063-1073.

Wang X, Sachs MB (1993) Neural encoding of single-formant stimuli in the cat. I. Responses of auditory nerve fibers. J Neurophysiol 70:1054-1075.

Winter IM, Palmer AR (1990) Responses of single units in the anteroventral cochlear nucleus of the guinea pig. Hear Res 44:161-178.

Yin TCT, Chan JCK (1990) Interaural time sensitivity in medial superior olive of cat. J Neurophysiol 64:465-488.

Yin TCT, Chan JCK, Irvine DRF (1986) Effects of interaural time delays of noise stimuli on low-frequency cells in the cat's inferior colliculus. I. Responses to wideband noise. J Neurophysiol 55:280-300. 